\title{
An Alignment-free Heuristic for Fast Sequence Comparisons with Applications to Phylogeny Reconstruction
}

\author{
Jodh Pannu \\ University of Central Florida \\ Orlando, FL, USA \\ jodh@knights.ucf.edu \\ Sharma V. Thankachan \\ University of Central Florida \\ Orlando, FL, USA \\ sharma.thankachan@ucf.edu
}

\author{
Sriram P. Chockalingam \\ Georiga Institute of Technology \\ Atlanta, GA, USA \\ srirampc@gatech.edu \\ Srinivas Aluru \\ Georiga Institute of Technology \\ Atlanta, GA, USA \\ srirampc@gatech.edu
}

\begin{abstract}
Alignment-free methods for sequence comparisons have become popular in many bioinformatics applications, specifically in the estimation of sequence similarity measures to construct phylogenetic trees. Recently, the average common substring measure, ACS, and its $k$-mismatch counterpart, $\mathrm{ACS}_{k}$, have been shown to produce results as effective as multiple-sequence alignment based methods in phylogeny. In this work, we present a novel linear-time heuristic to approximate $\mathrm{ACS}_{k}$, which is faster than computing the exact $\mathrm{ACS}_{k}$ while being closer to the exact $\mathrm{ACS}_{k}$ values compared to previously published linear-time greedy heuristics.
\end{abstract}

\section{KEYWORDS}

Approximate Sequence Matching; Phylogeny reconstruction

ACM Reference Format:

Jodh Pannu, Sriram P. Chockalingam, Sharma V. Thankachan, and Srinivas Aluru. 2018. An Alignment-free Heuristic for Fast Sequence Comparisons with Applications to Phylogeny Reconstruction. In $A C M-B C B$ '18: 9th $A C M$ Int'l Conf. on Bioinformatics, Computational Biology, and Health Informatics, Aug. 29-Sept. 1, 2018, Washington, DC, USA. ACM, New York, NY, USA, 1 page. https://doi.org/10.1145/3233547.3233648

\section{INTRODUCTION}

In this work, we present a novel greedy heuristic that is a more accurate approximation of $\mathrm{ACS}_{k}$ than [1]'s heuristic to estimate $\mathrm{ACS}_{k}$ and takes linear time. We also evaluate our approach using a dataset of mitochondrial genomes from 27 primates.

\section{METHOD AND RESULTS}

To approximate $\mathrm{ACS}_{k}$ of two sequences $\mathrm{X}$ and $\mathrm{Y}$, kmacs's heuristic uses the longest common substring of suffixes $X[i .$.$] and Y[j .$.$] as$ the initial anchor segment and performs a forward extension to identify a common substring with $k-1$ mismatches. Our algorithm is based on the observation that a $k$-mismatch common substring

Permission to make digital or hard copies of part or all of this work for personal or classroom use is granted without fee provided that copies are not made or distributed for profit or commercial advantage and that copies bear this notice and the full citation on the first page. Copyrights for third-party components of this work must be honored.

For all other uses, contact the owner/author(s).

ACM-BCB'18, August 29-September 1, 2018, Washington, DC, USA

(c) 2018 Copyright held by the owner/author(s).

ACM ISBN 978-1-4503-5794-4/18/08

https://doi.org/10.1145/3233547.3233648 of two suffixes $\mathrm{X}[i .$.$] and \mathrm{Y}[j .$.$] includes k-1$ common substrings separated by $k$ mismatch characters. Our linear-time heuristic is computed by extending, both forwards and backwards, all the $k-1$ matching substrings that overlap the position $i$, as anchors.

In order to evaluate the runtime, the accuracy and the effectiveness of our algorithm, we used the Primates dataset, which includes 27 primate mitochondrial genomes with a total length of $\approx 450$ kilobases. In addition to our method, we also ran kmacs and ALFRED-G [2] on a machine with Intel i5-6200 2.3GHz CPU and $8 \mathrm{~GB}$ of main memory. Figure 1 shows that our method's error percentage is lower compared to kmacs, but in terms of runtime, our method runs up to 2.5X faster than that of ALFRED-G. Using the

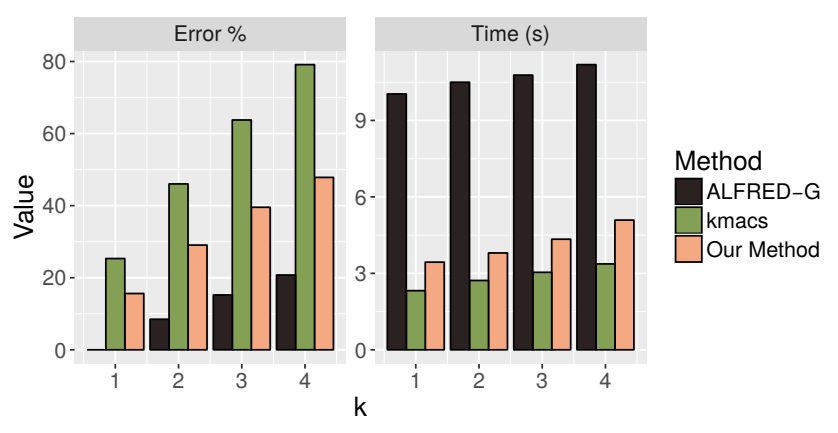

Figure 1: Average error \% of estimated $\mathrm{ACS}_{k}$ w.r.t. the exact $\mathrm{ACS}_{k}$ values and the runtimes for the Primates dataset.

PHYLIP software package, we also constructed phylogeny trees for the Primates based on the estimated $\mathrm{ACS}_{k}$. Results show that as evaluated by the Robinson-Folds distance, the trees constructed using our method is more accurate than kmacs, while being competitive in runtime compared to kmacs and much faster than ALFRED-G.

\section{REFERENCES}

[1] Chris-Andre Leimeister and Burkhard Morgenstern. 2014. Kmacs: the k-mismatch average common substring approach to alignment-free sequence comparison. Bioinformatics 30, 14 (2014), 2000-2008.

[2] Sharma V Thankachan, Sriram P Chockalingam, Yongchao Liu, Ambujam Krishnan, and Srinivas Aluru. 2017. A greedy alignment-free distance estimator for phylogenetic inference. BMC bioinformatics 18, 8 (2017), 238. 